\title{
Ruptura de cisto aracnóideo intracraniano causando higroma e hemiplegia súbita
}

\author{
Maurus Marques de Almeida Holanda*, Ana Moema Pereira da Nóbrega**, Stênio \\ Abrantes Sarmento*, Ussânio Mororó Meira*, José Alberto Gonçalves da Silva*** \\ Instituto de Neurologia e Neurocirurgia da Paraíba (INNPAR)
}

\section{RESUMO}

Os autores relatam dois casos de ruptura de cisto aracnóideo, sem história de trauma craniano, com formação de higroma, manifestando-se com quadro clínico de síndrome de hipertensão intracraniana e evoluindo com hemiplegia súbita.

São discutidos a etiologia, as manifestações clínicas, o mecanismo de ruptura do cisto e as imagens radiológicas que traduziram a evolução do evento.

\section{PALAVRAS-CHAVE}

Cisto aracnóideo. Higroma subdural.

\section{ABSTRACT neuroimaging are discussed. \\ KEYWORDS \\ Arachnoid cyst. Subdural higroma.}

Subdural higroma and acute hemiplegia due to non traumatic rupture of arachnoid cyst The authors present two cases of rupture of arachnoid cyst, whithout head trauma history, with formation of higroma, which presented as intracranial hypertension syndrome and subtle hemiplegia. The etiology, clinical manifestation, possible mechanisms of cyst rupture and the diagnostic

\section{Introdução}

Os cistos aracnóideos são coleções de líquido, envoltos por uma membrana que se assemelha à aracnóide, podendo se desenvolver em qualquer sítio do espaço subaracnóideo, ao longo do eixo cerebroespinhal. Sua localização mais freqüente é na fissura silviana. São diagnosticados na infância ou na idade adulta, podem atingir grandes dimensões e são clinicamente silenciosos. Há controvérsias quanto à sua etiologia e história natural, sendo descritas diferentes hipóteses, como agenesia de estruturas cerebrais, aracnoidite, secreção ativa de fluidos e mecanismo valvular $^{2,14}$. Poucos casos são citados na literatura que evidenciam a possibilidade de ruptura espontânea do cisto $^{10}$, ou por traumatismo ${ }^{19}$. Por outro lado, chamamos a atenção de dois casos de ruptura de cisto aracnóide com formação de higroma e hemiplegia súbita. A primeira suspeita clínica, em ambos, foi a de um quadro de acidente vascular cerebral.

\section{Relato de casos}

Caso 1: paciente do sexo masculino, com 82 anos de idade, em 28 de junho de 2000, após acesso de tosse, apresentou tontura intensa, seguida de perda da consciência. Foi avaliado por neurologista que, não observando déficit motor focal, prescreveu carbamazepina $200 \mathrm{mg}$ duas vezes ao dia. Em 17 de julho de 2000, foi admitido em hospital da cidade, apresentando crises generalizadas tônico-clônicas de difícíl controle, torporoso e hemiplégico à esquerda. A tomografia

*Neurocirurgião do INNPAR.

** Neurologista do INNPAR.

***Neurocirurgião do Hospital Santa Isabel. 
computadorizada (TC) de crânio evidenciou presença de cisto aracnóideo temporal direito e coleção subdural temporoparietal homolateral (Figura 1). O paciente foi submetido a cistoperitoneostomia, com interposição de válvula de baixa pressão, recebendo alta hospitalar, após um mês de internação, com hemiparesia esquerda. O controle tomográfico pós-operatório mostrou a resolução da coleção (Figura 2).

Caso 2: paciente do sexo masculino, com 61 anos de idade. Em 14 de junho de 2000, foi admitido em hospital da cidade com queixas de cefaléia holocraniana moderada e contínua, seguida de hemiplegia esquerda súbita, a qual evoluiu para hemiparesia após 3 horas. Não havia história pregressa de traumatismo cranioencefálico ou infecção do sistema nervoso central. A TC de crânio evidenciou a presença de cisto aracnóideo temporal e coleção subdural temporoparietal direita, com desvio da linha média para esquerda (Figura 3). Foi submetido à marsupialização do cisto, por via endoscópica, e apresentou fístula liquórica de alto débito após 15 dias, quando foi implantada válvula de baixa pressão, com boa evolução.

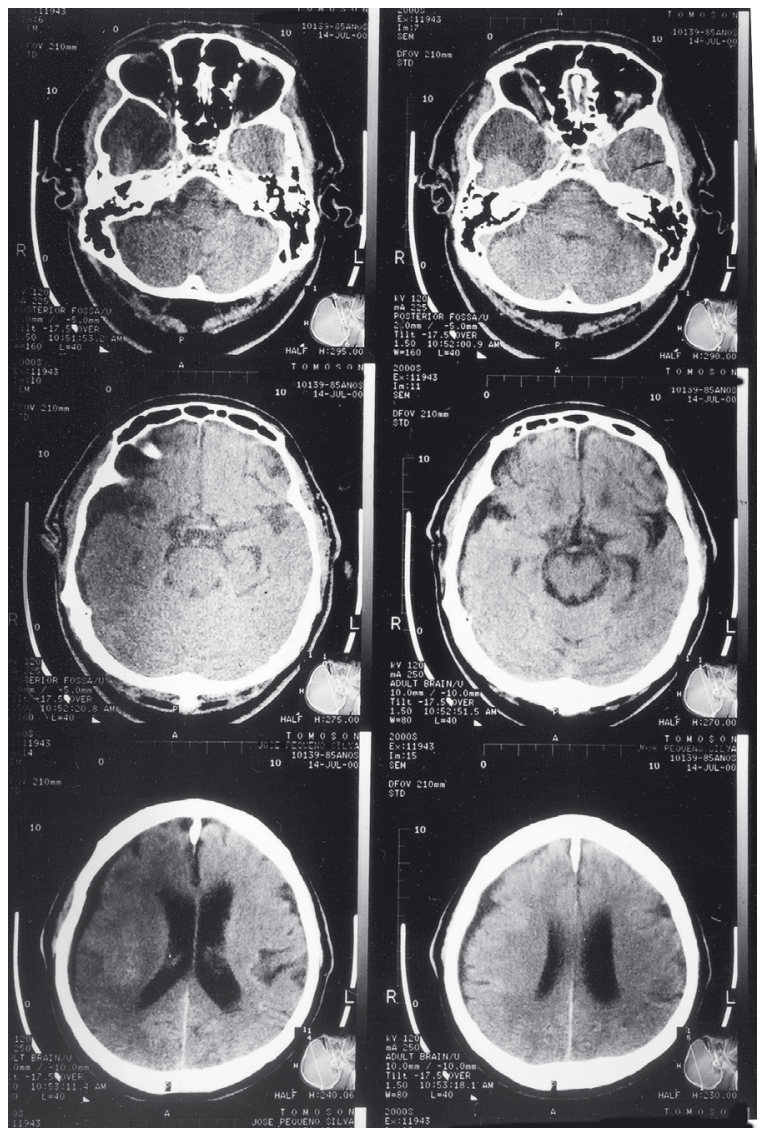

Figura 1 - TC mostrando cisto aracnóideo temporal direito e coleção subdural (caso 1).

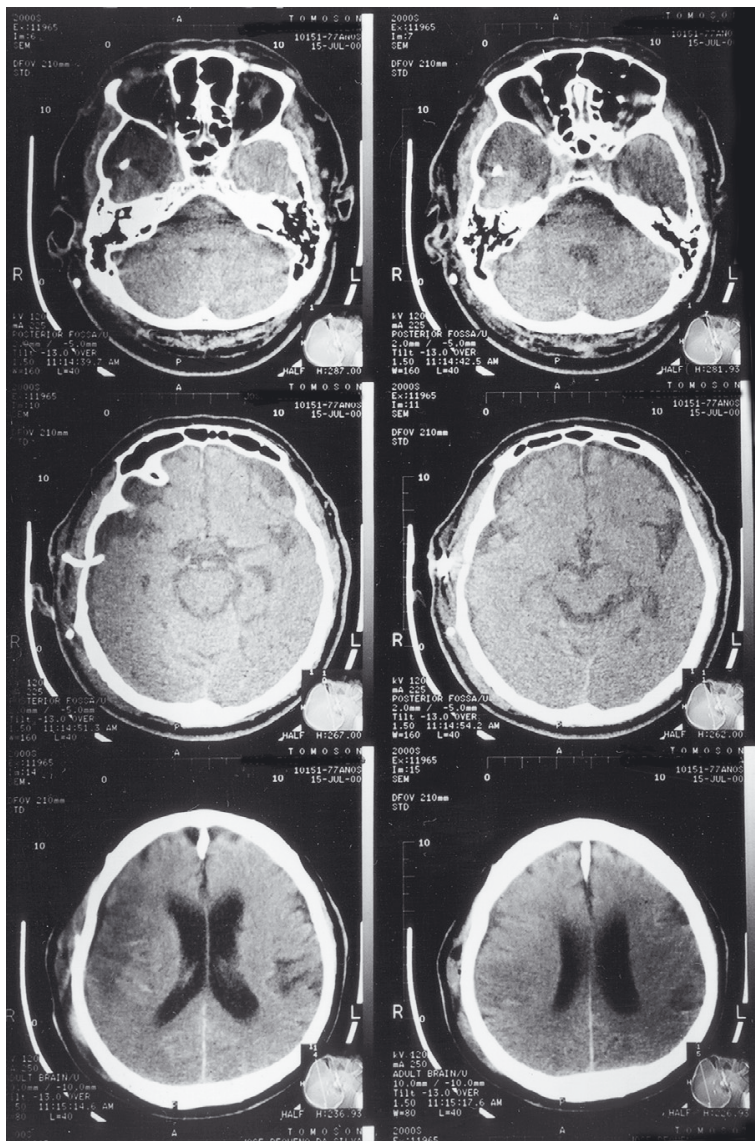

Figura 2 - TC após derivação cistoperitoneal (caso 1).

\section{Discussão}

Os cistos aracnóideos são relativamente raros e representam, aproximadamente, $1 \%$ das lesões intracranianas não traumáticas que ocupam espaço ${ }^{17}$. Com a introdução da TC e ressonância magnética (RM), tornaram-se comuns os achados dessas lesões, mesmo em pacientes sem sintomatologia relacionada assintomáticos.

Quanto à evolução natural, alguns cistos podem permanecer assintomáticos por toda a vida, enquanto outros podem se manifestar com sinais de hipertensão intracraniana, crise convulsiva, afasia motora, retardo neuropsicomotor, aumento do perímetro encefálico, abaulamento localizado do crânio, déficit focal motor e quadros clínicos distintos, dependendo da localização do cisto $^{1,4,5,8,110}$. De acordo com Wester ${ }^{22}$, os cistos aracnóideos são mais freqüentes em homens que em mulheres, na razão de $3: 1$, e ocorrem mais comumente na fossa craniana esquerda; $50 \%$ a $65 \%$ dos cistos estão localizados na fossa craniana média, $10 \%$ na região supra-selar e lâmina quadrigeminal, $5 \%$ na região frontal e $8 \%$ na fossa posterior ${ }^{15}$. 


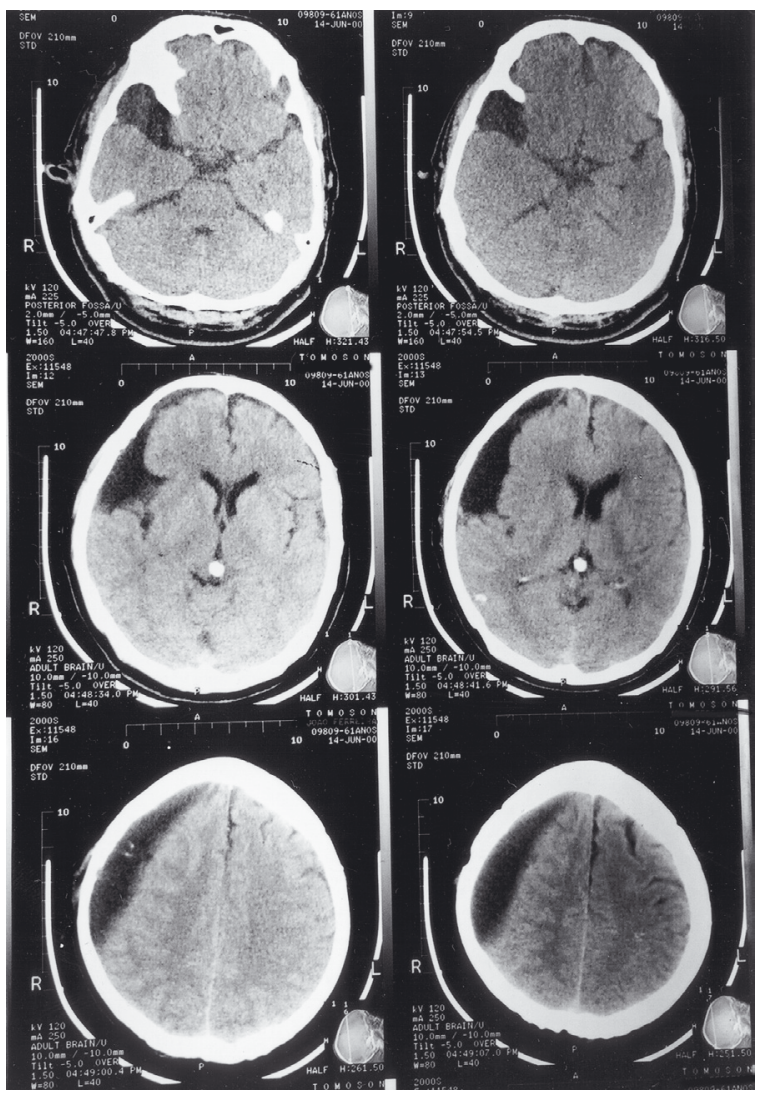

Figura 3 - TC mostrando o cisto aracnóideo, a coleção subdural e o desvio para a esquerda (caso 2).

Na etiopatogenia, a hipótese de agenesia de estruturas encefálicas foi sugerida por Robinson ${ }^{16}$ e defendida por Kato e cols. ${ }^{12}$ Esses autores consideravam os cistos da fissura de Sylvius secundários à coleção de líquido cefalorraquidiano (LCR) e associados à agenesia parcial do lobo temporal. Essa hipótese tornou-se obsoleta, pois hoje se sabe que o lobo temporal pode voltar a ocupar o seu espaço na fossa craniana média, após o tratamento adequado e mesmo espontaneamente, como descreveu Wester e cols. ${ }^{21}$.

Há autores que confirmam a formação de cistos secundários a aracnoidite decorrentes de infecções, traumatismos e hemorragias, que comprometem o espaço subaracnóideo ${ }^{18}$. Muitos deles consideram o cisto como uma duplicação primária da aracnóide. A razão por que os cistos se expandem tem sido objeto de debate durante a última década.

As duas hipóteses atualmente mais aceitas, para explicar a etiopatogenia desses cistos, atualmente, são a da secreção ativa de fluidos pela parede do cisto e a do mecanismo valvular. A primeira explicaria o crescimento dos cistos não comunicantes. Isso foi confirmado por Go e cols. ${ }^{8}$, que identificaram epitélio secretor em estruturas morfológicas da parede dos cistos.
Alguns autores demonstraram a comunicação dos cistos com o espaço subaracnóideo ${ }^{13,23}$, por meio de pneumoencefalografia e TC com cisternografia. Nesses casos, o crescimento do cisto ocorre por mecanismo valvular unidirecional e é influenciado pelas pulsações das artérias intracranianas e mudanças na pressão venosa $^{6,7,23}$. Esse mecanismo foi demonstrado na RM e confirmado por Santamarta e cols. ${ }^{17}$, durante procedimento endoscópico, ocasião em que foi visualizada e fotografada a presença de uma fenda na parede do cisto, durante os movimentos de abertura e fechamento $\operatorname{desta}^{7,17}$.

Nos dois casos apresentados neste relato, ora em estudo, é possível que tenhamos confirmado o dia exato da ruptura do cisto, quando ocorreu a hemiplegia, flagrando-se a coleção subdural durante exame tomográfico. Após esse momento, o primeiro paciente melhorou das crises convulsivas e o segundo referiu alívio da cefaléia e encontrava-se assintomático após 24 horas. Tivemos dificuldade durante a marsupialização endoscópica do segundo caso, pelo motivo da vascularização abundante da cápsula, provocando sangramentos durante o procedimento e nos limitando à comunicação com a cisterna carotídea. Foi deixada derivação externa do cisto, pela qual houve saída de LCR pouco sanguinolento e com alto débito; a derivação foi retirada após 48 horas. O paciente retornou, duas semanas depois, com fístula liquórica de alto débito, quando optamos por implantar uma derivação cistoperitoneal.

Holanda e cols. ${ }^{10}$ publicaram um caso assintomático de cisto aracnóideo, associado à coleção subdural não traumática, cuja evolução foi favorável sem intervenção cirúrgica. Nesse caso, foram adotadoas os critérios clínicos descritos por Robinson ${ }^{16} \mathrm{e}$ Tortelly-Costa e cols. ${ }^{19}$, os quais desaconselham o tratamento cirúrgico em casos de cisto aracnóide assintomático. Desse modo, admitimos a possibilidade de ter havido absorção da coleção subdural através das vilosidades aracnóideas.

Isso não ocorreu nos dois casos estudados, provavelmente em decorrência do insucesso de não se ter conseguido a comunicação dos cistos com o espaço subaracnóideo. Em conseqüência, foram implantadas válvulas de baixa pressão, mesmo levando-se em conta a possibilidade de os pacientes se tornarem shunt dependentes. Arai e cols. ${ }^{1}$ trataram 77 pacientes com cisto aracnóideo da fossa craniana média com implantação de derivação ventriculoperitoneal com interposição de válvula de baixa pressão, dos quais sete apresentavam hemiparesia que regrediu após o procedimento.

Esses casos mostram a importância do tratamento precoce dos cistos aracnóideos para minimizar a presença do déficit motor, não esquecendo a possibilidade de marsupialização dos cistos aracnóideos 
para o espaço subaracnóideo por via endoscópica, ou mesmo introdução de cateter fenestrado neste espaço, como tem preconizado muitos autores $^{2,4,9,11,13,17,20}$, no intuito de promover o esvaziamento natural e a absorção do seu conteúdo líquido pelas vilosidades aracnóideas. Desse modo, evitam-se as eventuais complicações inerentes à interposição das derivações cistoperitoneais e grandes acessos para marsupialização ${ }^{10,14}$.

\section{Referências}

1. ARAI H, SATOK, WACHI A, OKUDA O, TAKEDA N: Arachnoid cysts of the middle cranial fossa: experience with 77 patients who were treated with cystoperitoneal shunting. Neurosurgery 39:1108-13, 1996.

2. BEHRENS P, OSTERTAG CB: Stereotactic management of congenital midline cysts. Acta Neurochir (Wien) 123:141-6, 1993.

3. BELTRAMELLO A, MAZZA C: Spotaneous disappearance of a large middle fossa arachnoid cyst. Surg Neurol 24:181-3, 1985.

4. CAEMART J, ABDULLAH J, CALLIAUW L, CARTON D, DHOOGE C, VAN COSTER: Endoscopic treatment of suprasellar arachnoid cyst. Acta Neurochir (Wien) 119:68-73, 1992.

5. DE VOLDER AG, MICHEL CH, THAUVOY C, WILLEMS G, FERRIĖRE G: Brain glucose utilization in acquired childhood aphasia associated with a sylvian arachnoid cyst: recovery after shunting as demonstrated by PET. J Neurol Neurosurg Psychiatry 57:296-300, 1994.

6. DU BOULAY GH, O'CONELL J, CURRIE J, BOSTICK $T$, VERITY P: Further investigations on pulsatile moviments in the cerebrospinal fluid pathways. Acta Radiol 13:496-523, 1972.

7. ENZMANN DR, PELC NJ: Cerebrospinal fluid flow measured by phase-contrast cine MR. AJNR 14: 13017, 1993.

8. GO KG, HOUTHOFF HJ, BLAAUW EH, HAVINGA P, HARTSUIKER J: Arachnoid cysts of the sylvian fissure. Evidence of fluid secretion. J Neurosurg 60:803-13, 1984.

9. HARSH GR, EDWARDS MSB, WILSON CB: Intracranial arachnoid cysts in children. J Neurosurg 64:835-42, 1986.

10. HOLANDA MMA, DA SILVA JAG, BRITO JCF : Ruptura de cisto arcnóideo com formação de higroma e resolução espontânea. Arq Bras Neurocir (São Paulo) 17:130-3, 1998

11. JONES RF, H.WARNOCK T, NAYANAR V, GUPTA JM: Suprasellar arachnoid cysts: management by cyst wall resection. Neurosurgery 25:554-61; 1989.

12. KATO, MY, NAKADA, ARIGA N., KOKUBOY, MAKINO H: Prognosis of four cases of primary middle fossa arachnoid cyst in children. Childs Brain 7:195-204, 1980.

13. PIERRE-KAHN, A, CAPELLE L, BRAUNER R, SAINTEROSE C, RENIER D, RAPPAPORT R, HIRSCH JF: Presentation end management of suprasellar arachnoid cyst. J Neurosurg 73:355-9, 1990.

14. RAIMONDI AJ, SHIMOJI T, GUTIERREZ FA: Suprasellar cyst: Surgical treatment and results. Childs Brain 7:5772, 1980.

15. RENGACHARY SS, WATANABE I: Ultra-structure and pathogenesis of intracranial arachnoid cysts. J Neuropathol Exp Neurol 40: 61-83,1981.

16. ROBINSON, RG: The temporal lobe agenesis syndrome. Brain 88:87-106,1964.

17. SANTAMARTA D, AGUAS J, FERRER E: The natural history of arachnoid cysts: endoscopic and cine-mode MRI evidence of a slit-valve mechanism. Minim Invas Neurosurg 38:133-7, 1995.

18. SOHLER MP, CABRAL MB, PINHEIRO, RSA,TEMPONI GM, NOVIS, SAP: Cisto aracnoide pós-traumático. Rev Bras Neurol 25:23-5, 1989.

19. TORTELLY-COSTA A, FREITAS MRG, SÁ RMS SILVA BF: Cisto aracnóideo intracraniano associado com higroma subdural. Arq Neuro-Psiquiatr 43:917, 1985.

20. WESTER, K: Arachnoid cyst in adults: experience with internal shunts to the subdural compartment. Surg Neurol 45:15-24, 1996.

21. WESTER K: Arachnoid cysts in adults: Experience with internal shunts to the subdural compartment. Surg Neurol 45:15-24, 1996.

22. WESTER K, GILHUS NE, HUGDAHL K, LARSEN JL: Spotaneous disappearance of an arachnoid cyst in the middle intracranial fossa. Neurology 41:9, 1991.

23. WILLIAMS B, GUTHKELCH AN: Why do central arachnoid expand? J Neurol Neurosurg Psychiatry 24:1085-92, 1974

Original recebido em junho de 2002

Aceito para publicação em setembro de 2002

\section{Endereço para correspondência:}

Maurus Marques de Almeida Holanda

Rua Santos Coelho Neto, 200/802

CEP 58038-450 - João Pessoa, PB

E-mail:maurus@zaitek.com.br. 